\title{
Emergence of Digital Twins - Is this the march of reason?
}

\author{
Shoumen Palit Austin Datta \\ Senior Member, MIT Auto-ID Labs \\ Research Affiliate, Department of Mechanical Engineering \\ Massachusetts Institute of Technology, Room 35-203, 77 Massachusetts Ave Cambridge, MA \\ 02139, USA \\ Senior Scientist, Medical Interoperability Program, MDPnP Laboratory \\ Massachusetts General Hospital, Harvard Medical School \\ Partners MGH Research Building, 65 Landsdowne St, Cambridge, MA 02139, USA \\ Managing Director, NSF Center for Robots and Sensors for Human Well-Being \\ School of Engineering Technology, Purdue Polytechnic, Purdue University \\ 156 Knoy Hall, West Lafayette, IN 47907, USA \\ shoumen@mit.edu, sdatta8@mgh.harvard.edu
}

\section{Letter from Academia}

\begin{abstract}
Multiple forms of digital transformation are imminent. Digital Twins represent one concept, where we may use tools and technologies to "map" data (bits) from objects (atoms). It is gaining momentum because the "map" can act as a "compass" to reveal the status of atoms (things, devices, components, machines, people), process visibility and real-time transparency. Adoption of digital proxies, or digital duplicates, may face hurdles due to lack of semantic interoperability between architectures, standards and ontologies. The technologies necessary for automated discovery are in short supply. Progress depends on the convergence of information technology, operational technology and protocol-agnostic telecommunications. Making sense of the data, ability to curate data, and perform data analytics, at the edge (or mist, rather than in the fog or cloud) is key to value. Delivering algorithm engines to the edge, are crucial for edge analytics, if latency is detrimental. The confluence of these, and other factors, may chart the future path for Digital Twins. The number of unknown unknowns, and the known unknowns, in this process, makes it imperative to create global infrastructures and organize groups, to pursue the development of fundamental building blocks. We need new ideas and research in new domains to generate creative and innovative solutions.
\end{abstract}

Keywords. Digital Economy, Digital Twins, IoT, Agents, AI, Analytics, Cognitive Firewall, Cognitive Compass

\section{Introduction}

Being Digital (Nicholas Negroponte, 1996) ${ }^{1}$ and When Things Start To Think (Neil Gershenfeld, 2000) ${ }^{2}$ introduced the public to the potential emergence and the rise of smart machines. About a decade later, Jeff Immelt of GE started to market these ideas 
in the minds and machines campaign, claiming a future where self-organizing systems, sub-systems, multi-component subunits and modular embedded code, shall define the next generation of adaptive intelligent machines. When can we expect that "next" generation to emerge?

In 1513, the discovery of the isthmus at Panama by Vasco Núñez de Balboa triggered the idea of creating a trans-oceanic canal. Francisco Lopez de Gomara suggested (in his book, in 1552) Panama, Nicaragua, Darien and Tehuantepec as choices for a canal. Not for another 300 years, not until the $19^{\text {}}$ century, would the canal building actually commence under the leadership of Ferdinand Marie Vicomte de Lesseps a French diplomat. Ferdinand de Lesseps (19 Nov 1805 to 7 Dec 1894) could not complete the Panama Canal and did not live to see the successful completion of the Panama Canal in 1914 by the US Army Corps of Engineers ${ }^{3}$.

Creating intelligent adaptive machines faces a similar uphill battle. Our optimism is not unfounded but it may be burdened by the dead weight of old technology. Paving the path for new theories, new concepts and new forms of connectivity, in engineering design, of future systems, may lead to intelligent (?) machines. A greater challenge may be introducing cognition in systems, due to our wobbly and poor understanding of what constitutes intelligence 4 (AI).

Companies, afraid to delve deeper, are retrofitting existing machines with sensors to designate them as connected. Attaching sensors supposedly makes them smart. Workflow on steroids is peddled as intelligence in analytics. Others are collecting and feeding big (noisy) data sets to existing software systems and claiming "cognitive software" in use. The tapestry of buzz words and patch-work of programs are introducing glaring gaps, generating errors, callous disregard for physical safety, and inept approach to cybersecurity 5 in general.

One reason for the confusion, perhaps, is our general inability to ask correct questions. These are some of the questions from the field. What machines, devices and systems may be built with the tools and technologies at hand? How should we build and use them? Do we really want to just connect everything to collect big volume of data? Is it really all about data? What is data curation? How can we teach machines to achieve specific goals? Are these the correct questions to ask? Are these the questions even worth answering?

The debate rages on about answers. These and other related questions may find some answers hidden in bio-inspired design principles. Progress in bio-MEMS, bio-NEMS and molecular machines ${ }^{6}$ coupled with biological mimicry and cybernetics ${ }^{7}$ are elements which may (?) converge with $\mathrm{AI}^{8}$ in an over-arching strategic ${ }^{9}$ plan. Integrating that plan to inform engineering design is the Holy Grail. The command, control, and coordination of bio-inspired engineering design requires hardwaresoftware synchronization, by design (not later).

Time-synchronized hardware-software integration is one hallmark of cyber-physical systems ${ }^{10}$ (CPS) which is the foundation of embedded ${ }^{11}$ systems. The concept of digital twins emerged from NASA. Time guarantee (concurrence) in embedded systems is critical for aero/astronautics. To advance the diffusion of the digital twin concept, we must adopt practices ${ }^{12}$ borrowed from $\operatorname{CPS}^{13}$.

The current advocacy to advance the principles and practice of digital twins, from 
manufacturing to healthcare, calls for connectivity, by design. Systems should be able to discover, inherit, evaluate and share intelligence across sub-components and coordinate to turn on/off modular code embedded in sub-systems. We may monitor, analyze, control units (PLC) and sub-units, in real-time ${ }^{14}$ (sensors, actuation) and visualize operations not only at the system level, on-site, but attempt to view the operation of the entire ecosystem ${ }^{15}$. The latter, may accelerate diffusion of digital transformation using a systems engineering approach.

\section{The Rationale for Digital Twins}

\subsection{Signal vs Noise - IoT vs Digital Transformation}

The term IoT may have been coined at the MIT Auto ID Center (1999), but the past, present and future ${ }^{16}$ concepts of IoT have been brewing for almost a century. Milestones include Isaac Asimov's "Sally" the fictional autonomous car ${ }^{17}$, Herbert Simon's seminal paper18 ("talk to the computer"), Hiroshi Ishii's idea of "Tangible Bits" (People, Bits and Atoms ${ }^{19}$ ), Mark Weiser's paper ${ }^{20}$ "Activating Everyday Objects" as well as the 1991 article (Scientific American) ${ }^{21}$ and the vision ${ }^{22}$ of the "networked physical world" by Sanjay Sarma ${ }^{23}$, David Brock and Kevin Ashton (2001). The IoT roadmap ${ }^{24}$ promises to be even more dynamic in the future and scholarly discussions, including one by Alain Louchez ${ }^{25}$ outlines the layers of influence, hence, the challenges ahead.

IoT is a digital-by-design metaphor and a paradigm for ubiquitous connectivity. The value proposition rests on proper use of plethora of tools and technologies that must converge to make sense of the data. The hypothetical transparency is of little use without the data of things, if we wish to profit from IoT applications. On the other hand, digital transformation is a cacophony of ideas open to innovation from wireless systems ${ }^{26}$ as well as broadband communication ${ }^{27}$ and forthcoming $5 \mathrm{G}$ for time critical ${ }^{28}$ operations. The latter, if combined with $8 \mathrm{~K}^{29}$ visualization, may catalyze robotic surgery. Masses may benefit from standard surgical procedures such as laparoscopic cholecystectomy, appendicitis and phacoemulsification (cataract removal).

CNC machines, ERP, Web 2.0, fixed-task robots are examples of waves of digital transformation in business. The 2012 proposal ${ }^{30}$ from Sanjay Sarma of MIT Auto ID Labs to pursue a Cloud of Things initiative resonated globally and the concept was promoted by others (Finland ${ }^{31}$, France $^{32}$ and South Korea ${ }^{33}$, to name a few). The next wave appears to be the transition from manufacturing products (items to be sold), to the creation of a service ecosystem, around the product, to sell service as a pay-per-use model. Digital transformation includes establishing a digital leash to monitor, promote, connect, track and trace, in order to monetize every point of contact in the relationship (digital CRM), not only once (sales of product), but over the life time of the customer. Hence, product lifecycle management evolves to digital PLM with quality of service (not product delivery) as the KPI, and monetization tool. Quality of service (QoS) emerges as the readiness metric to gauge customer satisfaction. If QoS metrics are maintained by provider(s) or manufacturer(s), then the client or customer is expected to pay for the QoS level associated with the product-service, per contractual agreement, 
as long as the customer is consuming the benefit.

In instances where the product is not an object (eg, teleco) the business models are inextricably linked to "outcomes" the customer expects. Monetization of digital transformation from an outcome-based model is complex, due to the ecosystem of players and alliances. It is not easy to optimize and arrive at the point of convergence, to deliver the outcome as a seamless function, which involves end-to-end value chains, operating as a pre-agreed platform.

The task associated with monitoring each instance of engagement for micro-revenue collection and its disbursement is complex. We need to track each instance, and maintain a record of connectivity, in an irrefutable evidence log (eg blockchain $\left.{ }^{34}\right)$. The latter may act as a digital ledger ${ }^{35}$ to validate fractional micro-payments, due from each point of contact (PoC). The digital id of the service, delivered at the PoC, identifies the member of the supply chain providing the unit of service, at that specific instance. The latter may be a part of the sum of services, in the portfolio, that defines the service, and each may be weighted by a QoS the customer expects. The customer pays for the final outcome (value in the value chain). The sum of the parts must be delivered before the value perishes. The duration of that value may be widely divergent (compare retail vegetables in a grocery store vs data to predict risk of diabetes).

Synthesis of the parts to act as a seamless function is the challenge. Who will build the parts of the platform, which will be sufficiently open, and interoperable, to connect with the innumerable end points, at the edge? Who will build the blocks for the digital modules? Who will build the blocks for the blockchains?

\section{$2.2 \quad$ Digital Twins}

\section{Scenario}

Schlumberger is monitoring a drill-head in operation, on a drilling platform, in Outer Hebrides to determine the MTBF (mean time between failure) metric, and trigger replacement, to prevent work stoppage on the rig.

The camera at the tip of the drill-head, and drill-head (drill-case) sensors (eg, vibration, temperature, gyroscope, accelerometer) transmits (wired, wireless) video, audio and other data which must be analyzed as close to real-time as possible, with respect to object identification, precision geolocation and process linkage. AI(?) analytics updates MTBF metrics. Depending on MTBF range $(80 \%, 90 \%)$ as decided by business logic (when to replace), the drill-head spare parts supply chain (service, fulfillment) must be connected to auto-trigger the "head" when the MTBF range is reached. Purchase orders [supplier(s)] are followed by transport and logistics for delivery, and workforce scheduling, to execute the replacement prior to breakage (payment, contracts, invoices, and accounts payable, must be connected). Data about the drill-head, and lag time for each process/operation is captured by the operations management team, at a remote location, for future aggregate studies or collective evaluations. Can we visualize this entire end-to-end process as a Digital Twin operating in real time? 
In our current modus operandi this operation involves a plethora of operational silos (OT, drilling operation, mechanical engineering, systems, supply chain, finance, human resources), software (connectivity between different locations, cloud infrastructure, cybersecurity) and hardware (not only the spare parts and drill-head but also the computational hardware/servers at different locations which are essential for IT infrastructure).

The concept of DIGITAL TWIN posits that the flow of data, process and decision (this hypothetical scenario) is captured in a software avatar ${ }^{36}$ that mimics the operation or offers, at the least, a digital proxy.

The 3D "twin" or its digital proxy, may be visualized by an analyst or manager, on a location-agnostic mobile device (iPhone, iSkin). Drilling down on a schematic illustration with the word "drill-head" may link to the live video-feed from the drillhead camera which opens up on a new GUI (tab or window). Data fields (features, attributes, characteristics) related to the drill in operation (pressure, torque, depth, temperature, rotations) are visible by clicking on the icon for the drill. A plot showing the data, approaching the MTBF metric, may be instantiated using a command (icon "plot data"). It shows how the live sensor data is feeding the dynamic plot, displaying the characteristics of the drill-head, and the rate at which it is approaching the MTBF. The range may be set by humans (or the system), using prescriptive and/or predictive values based on "learned" values from machine learning tools operating in a "diagnostic" mode.

Will it allow for "what if" analysis? If the analyst viewing the Digital Twin, wishes to change the MTBF range and explore how the downstream processes may change (see principles of http://senseable.mit.edu/). The digital proxy for supply chain should spring into action, displaying delivery lag times from different suppliers, and cost of normal vs expedited delivery. The material composition of the alloy used in manufacturing the drill-head should be visible. The analyst may use an ad hoc selection process and identify a new vendor. Can the system trigger process workflow to alert the people (roles) along the way to clear the requisition, and generate purchase order for the new supplier? Can it auto-verify the new supplier to check credentials, inventory, cost, transportation scheduling, quality of service reports and customer reviews of prior contracts?

We are still on the mobile device or laptop with the Digital Twin app or its digital proxy. We watch the drill-head in action and a window displays the real-time data/analytics approaching MTBF. Using a different app, we identify a supplier to custom-design and 3D print-on-demand a drill-head with precision fit (think prosthetics). The supplier (www.quickparts.3dsystems.com/social-solutions/) downloads the video feed (from the cloud) of the drill-head operating in Outer Hebrides. The manager monitoring the end-to-end chain [a] selects the team of engineers who will replace the 3D printed drill using a HR menu which lists skill sets, proficiencies and years of expertise by category [b] pre-sets the command on the digital twin to actuate the replacement supply chain process, when MTBF reaches $72 \%$ because fulfilment takes 21 days, and by then (that is, 21 days later) the MTBF is predicted to reach $85 \%$ (code red - replace).

Each sub-unit provider must collaborate and synchronize (systems, standards, semantic interoperability) their role in the operation, and the representation of their function in 
the digital proxy, or in the digital twin model, in near real-time. The plethora of system providers, suppliers, third party software, analytics, cloud storage, fog providers and hardware component manufacturers - also - expects to be paid for the outcome, desired by the company (customer).

The design of content, and connectivity between such vast system of systems, calls for new principles of model-based systems engineering, to integrate global standards, to anchor architecture ${ }^{37}$ responsible to drive the digital by design paradigm. New models must inculcate the IoT digital-by-design metaphor with respect to connectivity by design, interoperability between standards by design, and cybersecurity by design. The silos of OT, IT and telecommunications must converge, to create this new digital-bydesign foundation for digital proxy and/or digital twins (digital proxy plus 3D models). In this paradigm shift, objects and things may not be baptized after birth to follow the digital persuasion, but will be born free of analog baggage. It may not need a path to digital transformation because they will be born digital.

Configuring Digital Twins: Creating the Blocks - Blockchain Paradigm? Lessons from cyber-physical systems (CPS) with respect to operational time synchronization may be key for certain forms of architecture for digital twins. Without open repositories, the process of creating (building) digital twins and the adoption of digital twins may be restricted to an industrial oligopoly. The vast majority of users cannot deploy an army of engineers to create custom digital twins for their exclusive experiments.

Rapid diffusion of digital twins calls for open source entity level models of subcomponents (units). Think of each SKU listed in a BOM (bill of materials), as a system made up of sub-systems. Next, imagine each sub-system with unit parts to serve as the "block" or base level unit which needs to be created (built). The "old world" notion may have stopped at the physical manifestation - the actual unit made of tangible material. In a digital twin world, we need a digital version.

In the era of digital twins, we will call on the source, that is, the CAD/CAM model owner of that unit, to create and contribute to a common repository (?) the software representation of the unit, replete with the physics of the material, and the engineering characteristics of its operational function. For example, the physics of the part will inherit natural laws which governs all entities. For example, if a spare part were to fall off a table (on this planet), it will fall $\underline{\text { down}}$ at a rate defined by the acceleration due to gravity of $9.8 \mathrm{~m} / \mathrm{s}^{2}$. The latter is an inherited ${ }^{38}$ attribute from the laws of physics (characteristics which forms the base in a 'layer cake' model).

To the informed mind, it is clear, we have encountered and entered the domain of semantics and ontologies.

The digital twins of the granular units (parts), to be useful, must be connected by their relationships to the relevant data feeds from sensors/gateways. These entity relationship models and parts (connectivity) must be accessible to managers or analysts who can drag and drop the parts from the repositories on a "sense" table (device GUI). The ontology of entity level relationship models for digital twins may use bio-inspired principles. Elements ${ }^{39}$ from disease models, for example, a bio-surveillance model, is shown below in Figure 1. 


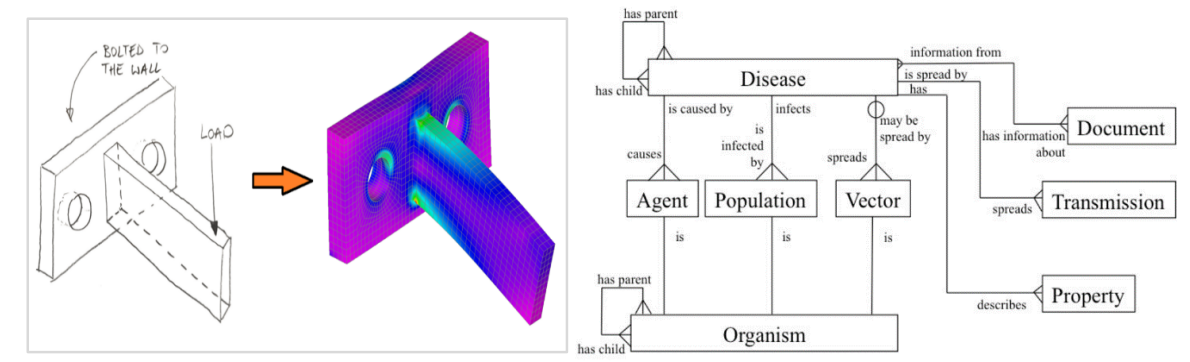

Fig. 1 - Digital twins as models of reality (illustration on left from $\left[{ }^{1}\right]$ and right from $\left[^{2}\right]$ )

To the untrained eye the cartoons may not suggest the cryptic complexity that must form their foundation. These foundations are "layer cakes" (for example, TCP/IP) which must be able to communicate with other "layer cakes" (for example, semantic $w_{e} b^{40}$ ) built on other principles, concepts or ontological frameworks. It is imperative we minimize the number of such architectures in order to fuel interoperability between architectures (requires interoperability between standards, access to open data dictionaries and shared ontologies).

The abstraction of the building blocks necessary for the digital twin movement may be similar, in principle, to the building blocks necessary to implement the use of blockchains, as a trusted digital ledger of connected instances. Who can we trust to build the blocks? This question is critical to practitioners of digital twins and blockchains. Both these concepts share homologies with IoT as a digital-by-design metaphor. The "block" in IoT may be the integrated platform, synthesized from subunits or blocks, containing data, of things.

The rate limiting step, which defines the functionality of all of the above, is inextricably linked with, and driven by the principles and practice of connectivity. In order to deliver value, connectivity must span a broad spectrum of dynamic ecosystems. Implementation of such connectivity must be protocol-agnostic, location agnostic and time sensitive (maximize transmission, minimize steps), with respect to "sense and response", between the edge and the core. Since IoT is expected to connect trillions of things, scalability will be a key enabler.

Have we encountered such "block" and "connectivity" concepts elsewhere? The common answer is Agent 41 systems. Marvin Minsky's brain connections related abstraction 42 "cube on cube" illustrates this concept where each cube is a software Agent. It is relevant to this topic because each cube may be viewed as a "block" in the blockchain or a baseline 'unit' in the digital twin paradigm (digital proxy of physical entity). The origin of the concept from software Agents, emphasizes the link to semantics, ontology, and related roots, which could go as deep as neural networks, cognition and even epistemology.

$\left.{ }^{1}\right]$ https://enterfea.com/wp-content/uploads/2017/04/A0-4.png

[ $\left.{ }^{2}\right]$ https://arxiv.org/ftp/arxiv/papers/1609/1609.05774.pdf 


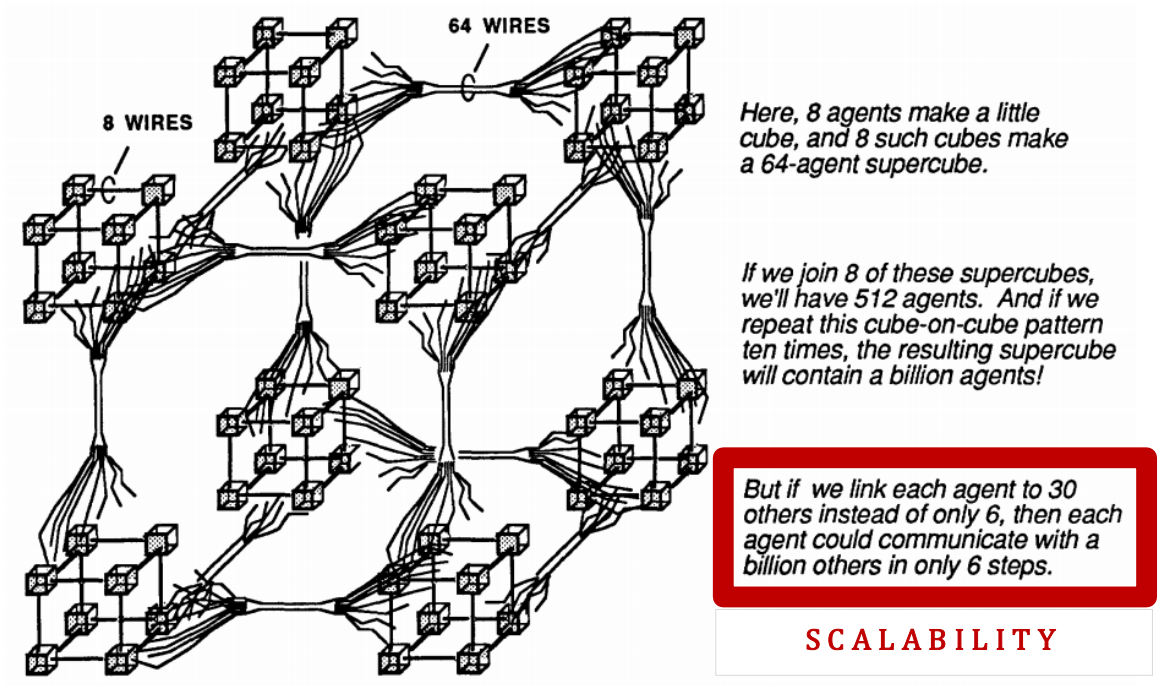

Fig. 2 - Illustration from page 315 (Appendix: Brain Connections) from Society of Mind by Marvin Minsky. MIT, 1985

The illustration (cube on cube) simplifies Minsky's abstraction and the principle of "blocks" to represent objects, data, process and decisions (outcomes). The blocks, when connected, can synthesize a variety of entities or networks ${ }^{43}$ joined by common digital threads. Alignment of appropriate blocks can lead to creating platforms necessary for implementation of IoT. Parts and sub-units, can be configured, to create a digital twin of a machine (drill-head). Instances and units of transactions, represented as blocks may constitute a digital ledger of events, similar to application of financial transactions using blockchain.

For a scenario at hand, please consider the act of driving your automobile (if you can still use a gas guzzler with an internal combustion engine ${ }^{44}$ ) to the proximity of a gas dispenser stand in a gas station (petrol pump).

Your car recognizes "arrival" at the gas station, correlates with low fuel reserve and unlocks the gas inlet. The petrol pump recognizes that your car is within the necessary proximity to the dispenser and recalls your choice for unleaded product. The nozzle from dispenser discovers the gas inlet, and commences fill-up, when your inlet allows, and confirms that the nozzle delivers petrol, not diesel. Once refueling completes, you see a green icon on your dashboard. You receive a SMS, indicating completion of fueling. The latter auto-triggered a financial transaction, to match the cost of fuel. Your bank confirms payment over iSkin or a smartphone app. It also informs your wife (authorized routing).

The convergence of IoT, digital twins and blockchain is evident. The ecosystem of enterprises, when dissociated by modular structures and associated by function, in an operational sequence, presents a series of steps, which can be sub-divided into "blocks" which are not only things/objects but software Agents, work units, process, authentication, authorization, decisions, outliers, feedback, security, metrics and dependencies. ${ }^{45}$ 


\section{Who will build these blocks?}
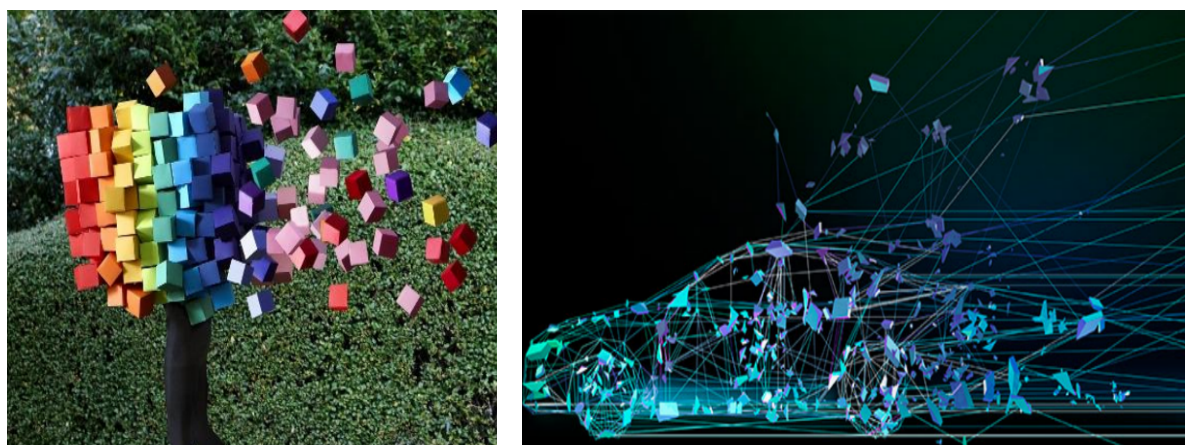

Fig. 3 - Blocks - a seamless operation represents an array of functions converging from diverse partner companies ${ }^{46}$ illustrations extracted from: left image from $\left[{ }^{3}\right]$ and right image from $\left[{ }^{4}\right]$

As it is with IoT, there will not be any one industry, or company, which may claim to be the front-runner. The modular building blocks, for the domains spanning and overlapping IoT, digital twins and blockchains (not limited to financial transactions) are quintessential to the global economy. The idea of a distributed team, or teams entrusted to architect these blocks, may seem reasonable. The fractured state of the world and the intrinsic impact of natural language (in)competencies on creating semantic dictionaries and ontological frameworks introduces severe socio-technical incongruencies. Hence, credible academic leadership of industry-government consortia, in partnership with global organizations or standardization bodies, may be a prudent option.

If a few global alliances create the blocks, and agree to establish the tools for interoperability, then we may anticipate a future global repository ${ }^{47}$ for these digital blocks to accelerate global digital transformation. The ubiquitous need for principles and practice of connectivity ${ }^{48}$ is salient to this discussion. The value expected from connectivity, assumes operation of multiple ecosystems, which must converge, to deliver the value. Table 1 suggests some of the layers, and components, necessary for this engineering ecosystem.

Local and global providers, who supply products and services germane to each layer (and several sub-layers within each layer), may not practice standard operating procedures (SOP). When volatility is the norm, it is wishful to expect SOP or expect groups in distant corners of the world to conform. The task of interoperability, and the ability to automate interoperability by "discovering" what is necessary to commence communication or cross-check resources, becomes pivotal. It is a tool which is not yet available. Do we need this tool to discover and replenish the gaps, for functional interoperability to commence?

[3] https://i.io.ua/img su/small/0010/63/00106374 n1.jpg

$\left.{ }^{4}\right]$ https://medium.com/vlb-token/blockchain-will-make-cars-safer-and-cheaper-bfda499a9f57 
Table 1. Layers, and components for this engineering ecosystem

\begin{tabular}{|c|c|c|c|}
\hline 01 & 1 & Infrastructure & Scaffolds which includes energy, internet engineering, telco networks \\
\hline 02 & $\mathrm{~T}$ & Telecommunications & Backbone of connectivity which enables location agnostic operations \\
\hline 03 & $\mathrm{P}$ & Protocol & Transaction triggered response operating agnostic of protocol-specificity \\
\hline 04 & $\mathrm{D}$ & Discovery & Blocks/entities must find each other in order to communicate (think RDF) \\
\hline 05 & C & Connectivity & Glue that enables digital transformation unless restricted by boundaries \\
\hline 06 & S & Sense & Data acquired from points of interaction to understand status / attributes \\
\hline 07 & $\mathrm{R}$ & Response & Analytics driven action/actuation based on integrating diverse knowledge \\
\hline 0 & 0 & Operate & Outcome as pre-determined or change direction if influenced by factors \\
\hline 09 & A & Adapt & Ability to remain dynamic and agile by recalibrating operations (eg SCM) \\
\hline 10 & $\mathrm{~K}$ & Knowledge & Learnings from operation (store/delete), dissemination, update analytics \\
\hline
\end{tabular}

It is expected that automating interoperability, may lead to auto-generation of APIs when interfaces "discover" that they cannot "talk" between models, data holders, tables, devices. It may trigger an automated mechanism to understand what needs to be understood between the systems, and then obtain the "glue" (for example, creates remote function call to source a "patch" from a repository) to facilitate interoperability. APIs are enablers of interoperability but creating API is not synonymous with instituting interoperability between systems. True interoperability involves the arduous task of semantic interoperability between systems, to facilitate data-driven multisystem performance optimization.

If someone speaks to me in Hebrew, I must know that I am listening to Hebrew before using Google to communicate in Hebrew. If I had a tool to auto-detect languages then it could help trigger $\left(\mathrm{CNN} / \mathrm{RNN}^{49}\right)$ Hebrew translation on my iPhone. An "avatar" or Siri or Cortana or Alexa, can then, guide my exchange.

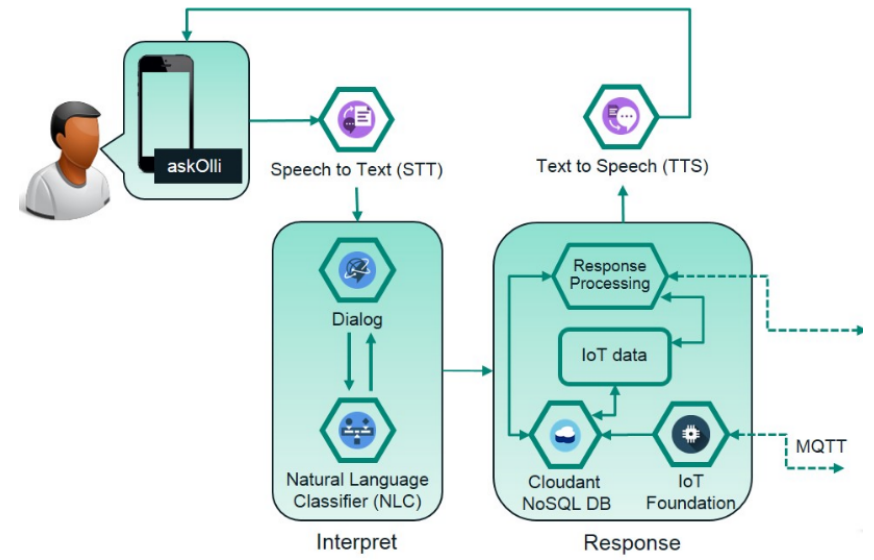

Fig. 4 - Watson Speech to text (STT) (illustration adapted from [5])

$\left[{ }^{5}\right]$ https://www.linkedin.com/pulse/meet-olli-fusion-autonomous-electric-transport-watson-kalyanaraman/ 
Discovery tools for auto-detection of attributes, and characteristics between entities, Agents, and models, are a core part of the digital-by-design metaphor, for example, IoT. Research in connectivity may help develop a new generation of digital semantic sensors to sense (what we may not know) what needs sensing. The concept of "sensing the need to sense" moves beyond semantic detection and introduces cognition. What we need is a cognitive compass.

The process of discovery, in addition to other elements, is a mix of semantic and cognitive modules. The inability to discover objects or identifying the wrong object or perturbing time-critical discovery (implants, medical devices) are potential pathways to compromising safety, security, privacy, authorization and cybersecurity. Intruders may be sophisticated to avoid semantic detection (5,50 and 500, are semantically, numbers, in different ranges). The importance of cognition and need to introduce cognitive firewalls 50 may be quintessential. Cognitive "supervisors" are necessary to keep an eye on semantic processes.

Thus, claims for ubiquitous computing, first, must find tools for discovery. The illusion of trillions of "things" connected via IoT are delusional and hyperbole marketed by uninformed publicists. Unless objects can safely discover each other, they may not connect (assuming that connectivity is protocol agnostic). Implementing tools and technologies ${ }^{51}$ central to discovery, and diffusion of cybersecurity as a service, may be required to accelerate digital transformation.

Digital twins may depend on safe "discovery" using semantic properties and cognitive rules for auto-configuration (think ad hoc auto-configuration of mesh networks) to create the desired assembly (machine floor, medical devices attached to patient, turbines, water purification). Digital twins will inherit attributes of the physical components and physics of the system. Ontology based semantic interoperability by design depends on entity level relationships. Distributed digital twins created by different sources, approved by cognitive supervisors, and connected by secure systems, may communicate, and form swarms, to help us make better decisions (one agent vs an agency or one ant vs a swarm of ants) employing the popular concepts of swarm intelligence..$^{52}$

Fraternal Twins: The First Bom - Digital or Physical? A century ago (1916), a theory about freemartins ${ }^{53}$ (the female of the heterosexual twins of cattle) generated interest about rare monozygotic twins ${ }^{54}$ in cattle. This led to the discovery of Müllerian Inhibiting Substance 55 (MIS). Pioneering research ${ }^{56}$ by Patricia Donahoe ${ }^{57}$ is beginning to unravel the role and therapeutic potential of MIS. It appears that the human genetic program is inherently female 58 (which came first - male or female, the chicken or the egg). If the fetus was left to differentiate without MIS, fetal development of müllerian ducts will produce a female child. That is, the "default" option of human genetics is programmed to produce females. Males exist due to inhibition of the development of müllerian ducts by MIS and differentiation of the Wolffian ducts by fetal testosterone. Females have evolved by design but males appear to be a modification or a by-product of evolution.

This very distant digression is intended to make the point that a fundamental plan, a base, exists in nature. The female plan is copied (duplicated) to produce the male, albeit, 
with modifications, catalyzed by a starter dose of MIS.

The concept of digital twins assumes we are creating a digital duplicate of the physical entity. But, the born digital metaphor may be, in reality, digital conception. In the industrial arena "things" may not be created without an engineering plan, technical specs and CAD/CAM models. The physical entity must be conceived as a digital model, before birth of the product. In the world of machines and parts, being conceived as digital is the design, to use a pre-natal metaphor. The physical entity is the post-natal stage, metaphorically. A plethora of exceptions may prove the rule. One such case are add-ons, for example, humans (and animals), attached to medical devices ${ }^{59}$ in homes or hospitals. We can create digital twins of this combined state, to monitor their physiology, but that "twin" or digital proxy may not be referred to as born digital. In this instance, for medical purposes, we can think of digital twins as a combinatorial outcome, for monitoring and acquisition of data. By closing the loop and enabling feedback from medical digital twins, we can save lives, thousands, or even millions, of lives. ${ }^{60}$

Think about a helicopter ${ }^{61}$ which may not be created as a physical entity unless we have a CAD/CAM (digital) version and create a simulation (using differential equations) to test the operation (rotation of the blades for lift-off). In recent models from manufacturers (Boeing 787), the pilot is subservient to the simulated model in the autopilot. An image conjured by the latter generated the apocryphal statement that in the airplane of the future there may be only two living creatures in the cockpit. A pilot and a dog. The role of the dog is to stop the pilot from touching the controls. The role of the pilot is to feed the dog.

If we reverse the logic of the digital twins we have discussed thus far, one might propose the digital blueprint as the primordial layer and the physical entity to be the fraternal twin (perhaps with limited mobility, if thinking about machines).

The digital blueprint and the simulated models ${ }^{62}$ which exists today, may be rapidly engineered with data feeds from the physical operation, to approach the "live" concept of digital twins. In proposing this modus operandi, we move closer to the domain of cyber-physical systems ${ }^{63}$. Time dependencies create the need for time guaranteed software ${ }^{64}$ which can understand the semantics of time and is protected from cyberthreats or time spoofing, by using cognitive firewalls, if time criticality is pivotal for its operation. For example, from $(\mathrm{t}=0)$ the decision to apply the brakes to the actual act of braking $(\mathrm{t}=\mathrm{n})$ to stop an automobile. 


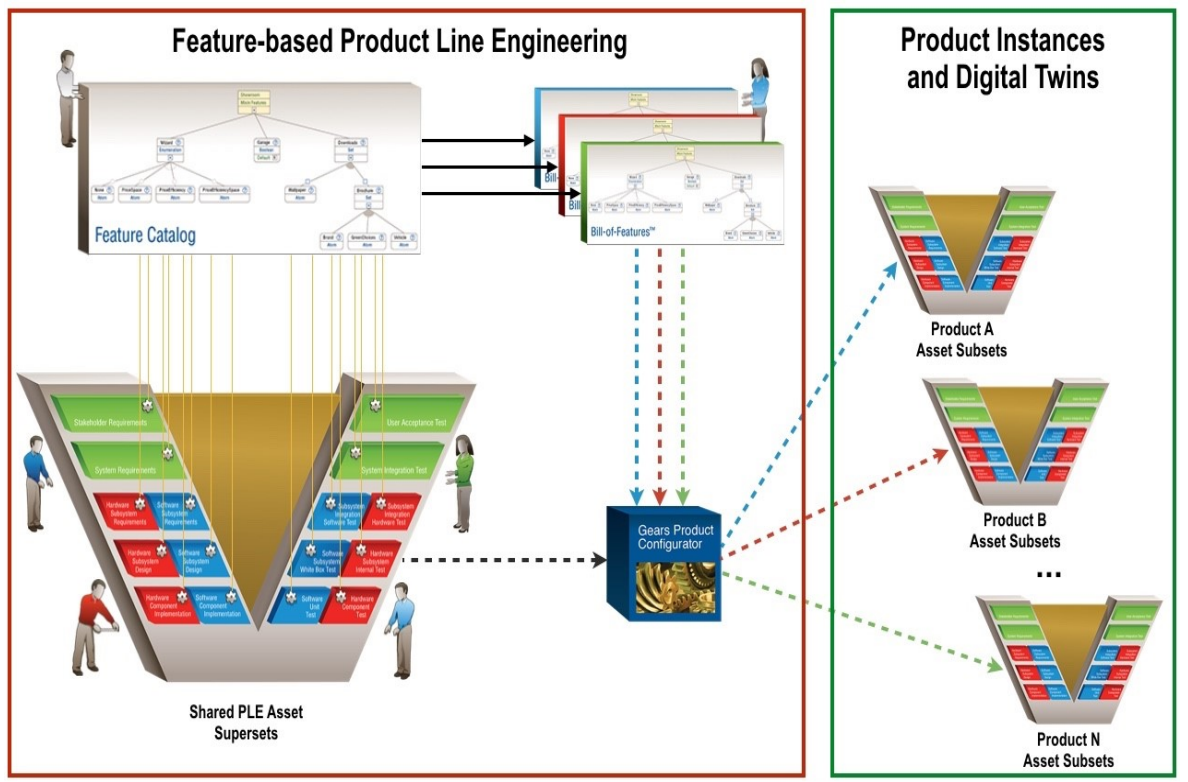

Fig. 5 - Feature Based Production Line Engineering (illustration extracted from [ $\left.{ }^{6}\right]$ )

The old world of engineering V models (Fig 5), spanning requirements, design, test, to user documentation. The upper tiers of the $\mathrm{V}$ represent the modeling and design phases where engineers build features based on product requirements, which flow to subsequent phases of the development, and manufacturing lifecycles. Individual features of a product are represented in the software 65 as pieces of modular source code that can be "turned" on or off, allowing assets to be generated based on a particular set of features (variant configuration). This accumulation of source code-based assets provides the foundation for creating physical products, but may be leveraged simultaneously to create a corresponding digital twin. Thus, the physical product is born digital. The digital twin can be bound using unique RFID identifiers, or other forms of component serial numbers, once the device is manufactured, and may operate through intelligent PLM platforms, throughout the product's life. The latter may ensure that the digital twin or the digital proxy, is auto-updated. Standards and certifications are key to adoption.

Confluence of Swarms Through Data Fusion - All Advantages Are Temporary. The digression about the conceptual see-saw, about whose twin is it anyway, is a thread of reasoning, not a barrier. It may make it easier to create the open repositories needed in the process of digital transformation. The road ahead for digital twins, digital proxies and digital transformation is fraught with problems and also brimming with potential. Driving fusions (please see illustrations below) through collaborative ecosystems may be one path to profit. There may not be a "winner takes it all" version in a cognitive digital twin economy.

$\left[{ }^{6}\right]$ https://www.slideshare.net/ArasPLM/product-line-engineering-meets-plm (slide 14) 

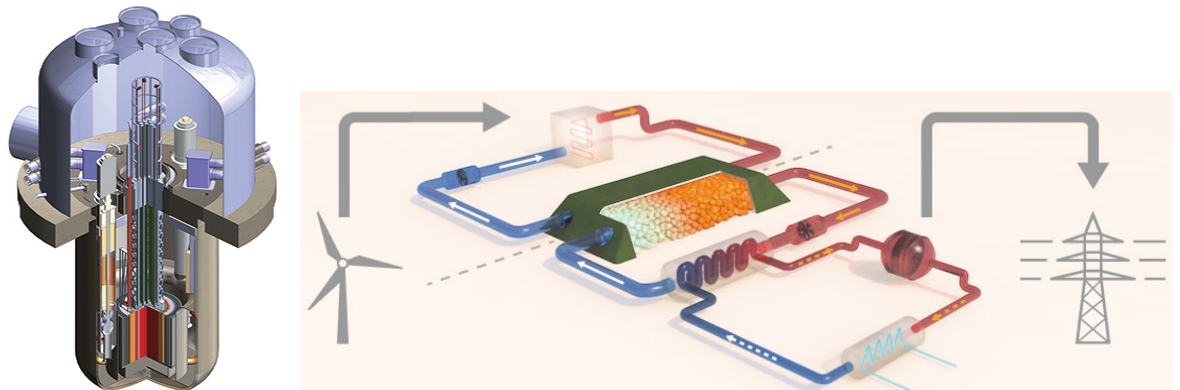

Fig. 6 - Digital Transformation: Energy Equilibrium - Elusive Quest for the Digital Mitochondria? To maintain homeostasis of energy production, distribution and load balancing. Adapting to multiple sources and types of energy obtained from diverse producers (domestic, commercial) with variable end points (homes, roadside charger, factories, mobile delivery). (illustrations extracted from: left image from [ $\left.{ }^{7}\right]$, right image from $\left[{ }^{8}\right]$ )

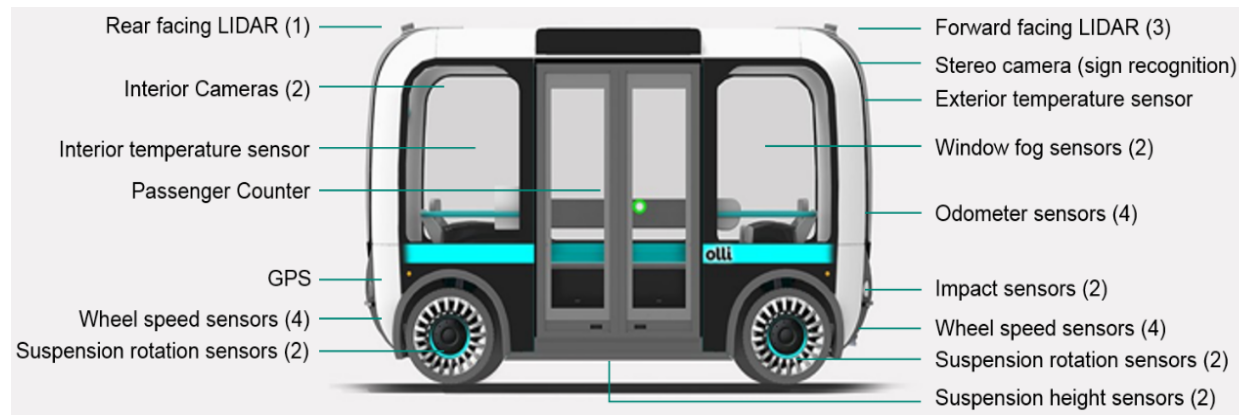

Fig. 7 - Digital Transformation: The Transportation Alloy - Alliance of Autonomy, IoT, Telecommunications and 3D Printing 66 (illustration extracted from [ $\left.{ }^{9}\right]$ )

To profit from fusion in the digital-by-design era, collaborative efforts ${ }^{67}$ may be one way forward. These examples of convergence (above) may be coupled with their operational digital twins or digital proxies. Information arbitrage from a wide crosssection of similar operations (from many devices) may provide a glimpse of patterns, which were previously unobtainable, due to the focus on one or few operations.

Hence, standards are key, followed by interoperability between standards and other facilitators (converters, adapters, translators, multi-homing) to increase connectivity and reduce incompatibility. The transaction cost may increase and reduce margin of profit, incur losses and downgrade the brand, if architectural and/or structural discrepancies, continue to pose barriers to function and/or outcome, expected by the customer.

A typical laptop deals with 250 compatibility standards. About $20 \%$ of the standards

$\left.{ }^{7}\right]$ http://www.world-nuclear-news.org/NN-TerraPower-brings-BandW-on-board-180214ST.html $\left[{ }^{8}\right]$ http://rief-jp.org/ct4/71847

[?] https://www.linkedin.com/pulse/meet-olli-fusion-autonomous-electric-transport-watson-kalyanaraman/ 
are from individual companies, while $44 \%$ are from consortia and 36\% from accredited standards development organizations. The complexity in laptops will be dwarfed by the variability expected for Digital Twins. But the use of ICE (Internalizing Complementary Externalities) like principles may evolve to create working solutions. ${ }^{68}$

Complementarity, compatibility and interoperability, assures us that we can expect visibility not only of one operation (which is what one industry or one team may monitor) but a group of hundreds or thousands of such operations. This massive data set may help us to understand patterns, predict faults, detect anomalies and use true "big" data, data curation and higher level metadata, to feed other functions, such as data driven policy, security threats and intruder detection using cognitive pattern analytics.

Consider the cartoon (below, left) of a physical event and assume that we have a digital twin of that operation that an analyst or manager can remotely view to "see" or monitor the physical operation in progress.

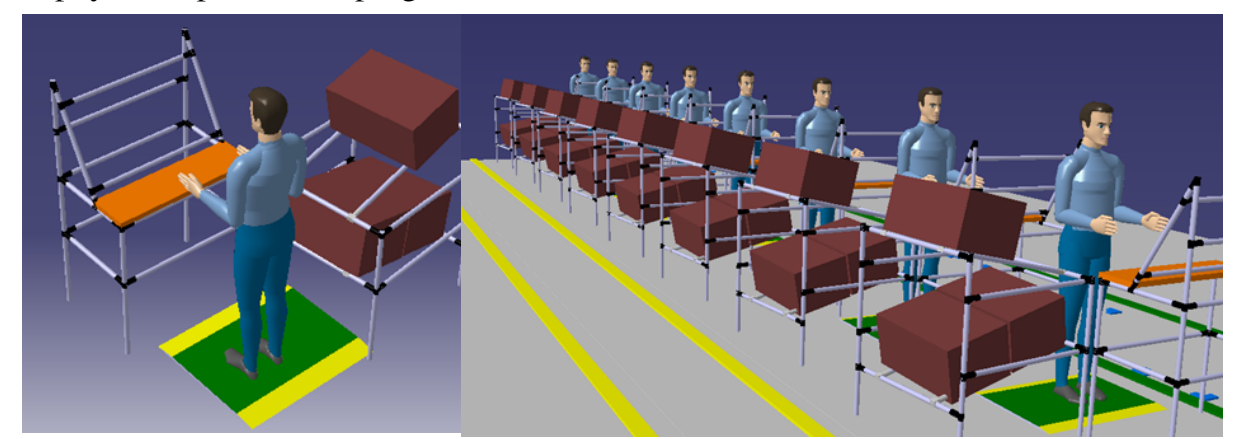

Fig. 7 - Physical System (cartoon adapted from illustrations by Lego®)

But, if the physical event (above, left) is not an isolated scenario, then digital duplication may generate (above, right) a form of digital transformation which may be representative of a digital swarm or flock. ${ }^{69}$

This digital vision of aggregated events, may generate big data and metadata, from precision patterns, which may be extracted or extrapolated with respect to process, performance and profitability. Any one instance may not offer sufficient incisive insight, but applying the principles of swarm intelligence to hundreds of instances, may provide wealth of information (not only data) which could enhance decision support systems and improve the monetization ${ }^{70}$ potential. 


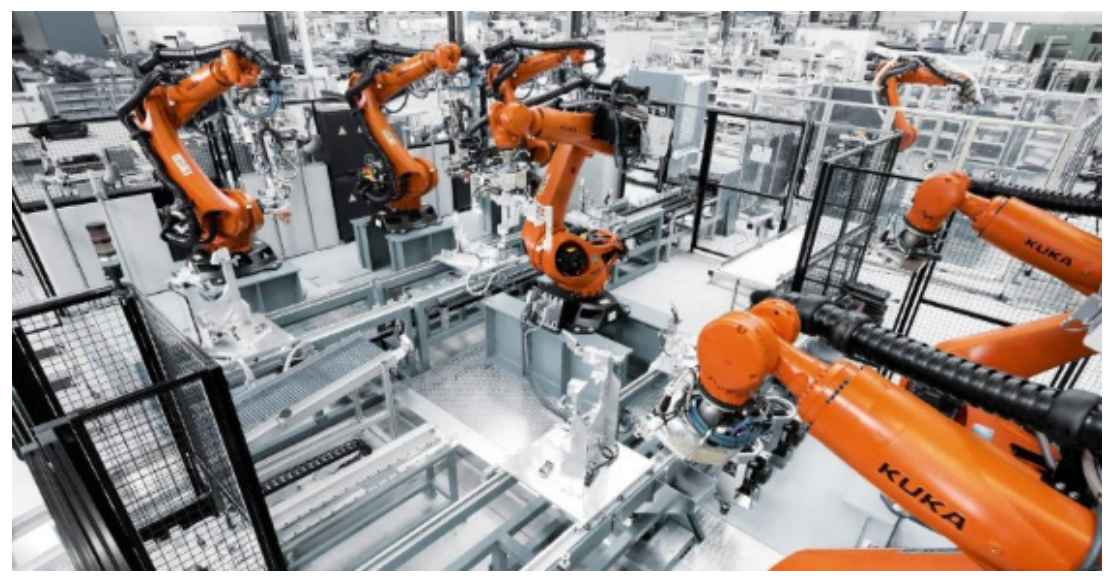

Fig. 8 - Robots (illustration extracted from $\left[{ }^{10}\right]$ )

Swarms of robots (networks, connected via combination of cloud, fog, mist) are likely to generate massive amounts of data. This data, if acquired, analyzed, and used in feedback control, may optimize process, reduce waste and compress production time. Use of "intelligent" decision systems platforms, various levels of automation, and predictive analytics, may transform the concept of 'zero' downtime to manufacturing reality, more or less.

Data from swarms may improve detection of anomalies, predictive analytics (if equipment needs a part or replacement) and errors or red herrings in the swarm may indicate security threat, breach or may elicit unusual activity alerts. The blockchainlike digital ledgers, in the backbone of the digital twins, may be useful in identifying the point of anomaly and associated objects or humans in the loop. Combined with advances in hack-proof ${ }^{71}$ code and cognitive firewalls, this approach may add a new dimension to systems cybersecurity.

The swarm and flock approach, if applied to the developing notion of smart cities, may offer relatively precise information, from digital twin operations of scale-free networks in urban digital transformation. Monitoring digital proxies of water valves, in operation, to control or regulate water waste, water security and water pollution. From an engineering systems point of view, the digital abstraction is applicable to city-level applications. Cities are inter-dependent cascade of systems and networks ${ }^{72}$ such as energy networks, traffic networks, sewer networks, communication networks, road networks and emergency response networks. The latter can make a difference between life and death.

The vision of network convergence may be crippled and remain impotent without architectures which are resilient, fault tolerant and uses standards which are dynamic. But, interoperability between standards are rather difficult when competition fuels mistrust, spurs acrimony and short-term profits are the life-blood of the industry. Digital transformations calls for confluence of ideas beyond the horizon and new roads to reach the luminous summit. Investment in scientific ${ }^{73}$ vision is often viewed with

$\left[{ }^{10}\right]$ https://www.autodesk.com/solutions/robot-programming-software 
reservation, excessive caution, undue skepticism and even disdain. The latter is most unfortunate for the progress of civilization.

Digital twins, IoT, blockchains, AI and swarm intelligence may re-define our imagination and future vision of globalization. That sense of the future requires businesses to re-think about ROI and profits, re-configure micro-payments and microrevenue models, but not at the expense of investment in $\mathrm{R} \& \mathrm{D}$. The latter is quintessential for innovation, and a tool to catalyze the principles of digital economics, to accelerate globalization.

In part, this idea originates from Marshall McLuhan ${ }^{74}$ that anyone, anywhere, may consume the same information. Digital Twins are another example of how this idea may be the reality for humans and machines, at multiple levels.

For example, a manufacturing plant in China, may be operating a component or machine sub-system, (atoms), which is represented by the Digital Twin or the data model of the digital proxy (bits). The global supply chain analyst, in India, monitors the status of the part via PLM. SCM intelligent decision support can trigger a replacement part from a supplier in Tampere, Finland or the component may be 3D printed by DDM Systems in Atlanta, GA and shipped to the factory in Dalian, China.

Taking this idea one step further, if the transport cost of bits approach zero, the transport to Dalian may be replaced, by sending the bits from the 3D architect in USA, to the 3D printer in Dalian, China, to 3D print the part. Digital Twins, through the process of digital transformation, shall lead us, perhaps, to the true digital economy, which once was an idea, implicit in the trade model, proposed by Paul Krugman ${ }^{75}, 40$ years ago.

\section{Conclusion}

At the dawn of the 21"Century, the internet was viewed by different groups to serve different functions. In some cases, it served as a storage platform ${ }^{76}$, others perceived it as a copying machine ${ }^{77}$, and economists ${ }^{78}$ explored its ability to reduce transaction cost (eg, communication, replication, transportation, tracking, verification, search) and democratization of information, as a catalyst for global economic growth.

Digital transformation, in the 21"Century, is the ability to represent atoms, in terms of bits. It stems from the seminal work by Claude Shannon ${ }^{79}$ which grew roots during the $20^{\text {m }}$ Century (Shannon information theory).

Digital transformation is made possible by the internet. Digital Twins and its "lite" version, digital proxy, are a part of the fabric of digital transformation, likely to affect most enterprises, worldwide, willing to duplicate the physical model for remote monitoring, viewing and controlling, based on a digital format (iPhone).

It is almost justified to think about the internet as a "giant copying machine" which can "copy" physical objects (atoms), to generate corresponding Digital Twins, a representation of information about the atoms, in terms of bits. Hence, we are not dealing with entirely new concepts, just new modes of expression.

Digital Twins are akin to the "emperor's new clothes" which are made of pre-existing conceptual yarns. We have added new vernacular and embedded the fabric with potentially new widgets (for example, the use of blockchain, as a verification tool). 
In this article, we call for an open source approach, to create the "blocks" or modules, necessary to democratize the ad hoc and en masse configuration of Digital Twins, by non-experts. The latter may no longer limit the use and application of Digital Twins in the hands of experts, alone.

Digital Twins may evolve as a tool, unconstrained by domains, beyond the boundaries of high performing economic regions, and contribute to economic growth, through open source platforms for digitization. Economic ${ }^{80}$ growth from such "digital dark matter" and other intangible benefits to the global economy, remains unmeasured. The impact from Digital Twins, digital proxies and digital duplicates, and cumulative benefits, may be only limited, by our imagination.

Industry must embrace change, imagine paths to reduce transaction cost, and shoulder the need to balance uncertainty, which may accompany the dynamics of digital economics. Leaders must proactively support the call for creating structures, necessary to pursue collaborative initiatives ${ }^{81}$ through investment in massive workforce development, skills training, digital learning ${ }^{82}$, education, research, institutional advancement and the pursuit of dignity.

\footnotetext{
${ }^{1}$ Nicholas Negroponte (1996) Being Digital, Vintage Books

${ }^{2}$ Neil A. Gershenfeld (2000) When Things Start to Think, Henry Holt and Company

${ }^{3}$ www.simonandschuster.com/books/The-Path-Between-the-Seas/David-

McCullough/9780743262132

${ }^{4}$ http://bit.ly/EYE-in-AI

5 http://www.politico.com/agenda/story/2015/06/internet-of-things-privacy-risks-security000096

${ }^{6}$ https://www.nobelprize.org/nobel prizes/chemistry/laureates/2016/popularchemistryprize2016.pdf

${ }_{8}^{7}$ http://uberty.org/wp-content/uploads/2015/07/Norbert Wiener Cybernetics.pdf

8 www.whitehouse.gov/sites/default/files/whitehouse files/microsites/ostp/NSTC/preparing for the future of ai.pdf

${ }^{9}$ www.whitehouse.gov/sites/default/files/whitehouse files/microsites/ostp/NSTC/national ai $\mathrm{r}$ d strategic plan.pdf

${ }^{10}$ www.ieeecss.org/sites/ieeecss.org/files/documents/IoCT-Part3-02CyberphysicalSystems.pdf

11 http://leeseshia.org

$12 \mathrm{https://cse.nd.edu/seminars/cse-seminar-series-rahul-mangharam}$

13 https://s3.amazonaws.com/nist-

sgcps/cpspwg/files/pwgglobal/CPS PWG Framework for Cyber Physical Systems Release 1 0Final.pdf

${ }^{14}$ Lee, E.A. (2015) The Past, Present and Future of CPS. Sensors 15 4837-4869

http://www.mdpi.com/1424-8220/15/3/4837

15 https://thingful.net/

${ }^{16} \mathrm{http}: / /$ www.computerworld.com/article/3076213/internet-of-things/the-iot-past-present-andfuture-an-interview-with-professor-sanjay-sarma.html

${ }^{17} \mathrm{https}: / /$ en.wikipedia.org/wiki/Sally (short story)

${ }^{18} \mathrm{http}$ ://digitalcollections.library.cmu.edu/awweb/awarchive?type=file\&item=34057

${ }^{19}$ Ishii, H. and Ullmer, B. (1997) Tangible Bits: Towards Seamless Interfaces between People, Bits, and Atoms in Proceedings of CHI'97 (ACM, March 1997)

http://tangible.media.mit.edu/person/hiroshi-ishii/

${ }^{20} \mathrm{http}: / /$ citeseerx.ist.psu.edu/viewdoc/download?doi=10.1.1.43.130\&rep=rep1\&type $=$ pdf

${ }^{21}$ https://www.ics.uci.edu/ corps/phaseii/Weiser-Computer21stCentury-SciAm.pdf
} 
${ }^{22} \mathrm{http}: / /$ cocoa.ethz.ch/downloads/2014/06/None MIT-AUTOID-WH-001.pdf

${ }^{23} \mathrm{http} / / /$ autoid.mit.edu/people-2

${ }^{24}$ https://www.technologyreview.com/s/601013/the-internet-of-things-roadmap-to-aconnected-world/

${ }^{25}$ https://www.ntia.doc.gov/files/ntia/publications/iot rfe june 2016 georgia tech cdait alain louchez.pdf

${ }^{26}$ https://www.whitehouse.gov/the-press-office/2016/07/15/fact-sheet-administrationannounces-advanced-wireless-research

${ }^{27}$ https://transition.fcc.gov/national-broadband-plan/national-broadband-plan.pdf

${ }^{28} \mathrm{http}: / /$ ucsdnews.ucsd.edu/pressrelease/keeping track of time in cyber physical systems

${ }^{29} \mathrm{http}: / /$ www.nhk.or.jp/8k/history/index e.html

${ }^{30} \mathrm{http} / / /$ news.mit.edu/2012/auto-id-cloud-of-things-big-data

${ }^{31} \mathrm{http} / / /$ ubicomp.oulu.fi/files/cscwd13.pdf

32 https://hal.inria.fr/hal-01004489/file/city06-petrolo.pdf

33 https://www.apan.net/meetings/Nantou2014/Sessions/IoT/Cloud-of-Things-APAN38-

2014.pdf

${ }^{34}$ http://bit.ly/Block-Chain

35 https://newsroom.accenture.com/content/1101/files/Cross-FSBC.pdf

${ }^{36} \mathrm{http}: / /$ www.zatar.com/blog/avatars-and-iot-architecture

${ }^{37} \mathrm{https} / / / \mathrm{hbr}$.org/2014/11/setting-standards-for-the-internet-of-things

${ }^{38}$ MPEG http://oxygen.csail.mit.edu/videosketching.html $\bullet$ CSAIL

http://oxygen.csail.mit.edu/Overview.html

${ }^{39} \mathrm{https} / / /$ arxiv.org/ftp/arxiv/papers/1609/1609.05774.pdf

${ }^{40}$ www.w3c.org

${ }^{41}$ Datta, S. (2002) Agents - http://dspace.mit.edu/handle/1721.1/41914

${ }^{42}$ Minsky, Marvin (1985), Society of Mind -

http://www.acad.bg/ebook/ml/Society\%20of\%20Mind.pdf

${ }^{43} \mathrm{http}: / /$ spectrum.ieee.org/computing/networks/metcalfes-law-is-wrong and

http://www.dtc.umn.edu/ odlyzko/doc/metcalfe.pdf

${ }^{44}$ http://www.spiegel.de/auto/aktuell/bundeslaender-wollen-benzin-und-dieselautos-ab-2030verbieten-a-1115671.html

${ }^{45} \mathrm{http}: / /$ esd.mit.edu/WPS/2007/esd-wp-2007-17.pdf and

http://dspace.mit.edu/handle/1721.1/41902

${ }^{46}$ http://qz.com/801640/darpa-blockchain-a-blockchain-from-guardtime-is-being-verified-bygalois-under-a-government-contract/

${ }^{47}$ www.scientificamerican.com/article/world-repository-of-human-genetics-will-move-toamazons-cloud/

${ }^{48}$ Principles and Practice of Connectivity (pdf of pptx)

https://dspace.mit.edu/handle/1721.1/104429

${ }^{49}$ https://dspace.mit.edu/handle/1721.1/101151 and https://arxiv.org/pdf/1411.4555v2.pdf as well as https://arxiv.org/pdf/1603.08270.pdf

${ }^{50} \mathrm{https}: / /$ dspace.mit.edu/handle/1721.1/104456

${ }^{51}$ http://oxygen.csail.mit.edu/videoh21 visit.html $\mathrm{l}$ and

http://oxygen.csail.mit.edu/videomultilingual.html

${ }^{52} \mathrm{http} / / /$ www.antoptima.ch/pdf/pr harvardbusiness $0105 . p d f$

${ }^{53} \mathrm{https} / / / w w w . j s t o r . o r g /$ stable/pdf/1641047.pdf

${ }_{55}^{54} \mathrm{http} / / /$ www.nature.com/hdy/journal/v2/n1/pdf/hdy19481a.pdf

${ }^{55}$ Price JM, Donahoe PK, Ito Y, Hendren III WH (1977) Programmed cell death in the Mullerian duct induced by Mullerian inhibiting substance. American Journal of Anatomy 149 353-375

${ }^{56}$ http://press.endocrine.org/doi/pdf/10.1210/edrv.22.5.0445 
${ }^{57} \mathrm{http}: /$ hsci.harvard.edu/people/patricia-k-donahoe-md

${ }^{58} \mathrm{https} / / /$ www.ncbi.nlm.nih.gov/pubmed/7828438

${ }^{59} \mathrm{http}: / /$ mdpnp.mgh.harvard.edu/

${ }^{60} \mathrm{http}: / /$ www.bmj.com/content $/ 353 / \mathrm{bmj} . \mathrm{i} 2139$

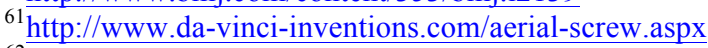

${ }^{62}$ https://www.modelica.org/

${ }^{6} \mathrm{https}: / /$ www.seas.upenn.edu/ rahulm/

${ }^{64} \mathrm{https} / / /$ www2.eecs.berkeley.edu/Pubs/TechRpts/2008/EECS-2008-72.pdf

65 http://www.biglever.com/extras/PLE LifecycleFramework.pdf

${ }^{66} \mathrm{http}: / /$ www.theverge.com/2016/6/16/11952072/local-motors-3d-printed-self-driving-buswashington-dc-launch

${ }^{67} \mathrm{https}: / /$ www.regulations.gov/contentStreamer?documentId=NHTSA-2014-0022-

$0001 \&$ attachmentNumber $=1 \&$ disposition $=$ attachment\&contentType $=$ pdf and

http://vistadataproject.info/

${ }^{68} \mathrm{http}: / /$ people.bu.edu/tsimcoe/documents/working/FourPaths.pdf

${ }^{69}$ Berrondo, M. and Sandoval, M. (2016) Defining emergence: Learning from flock behavior. Complexity 21 69-78 doi:10.1002/cplx.21711

${ }^{70} \mathrm{http}: / /$ www.icosystem.com/about-us/

${ }^{71} \mathrm{https}$ ://www.quantamagazine.org/20160920-formal-verification-creates-hacker-proof-code/

${ }^{72} \mathrm{http}$ ://journals.aps.org/prx/pdf/10.1103/PhysRevX.6.011036 and

http://dx.doi.org/10.1063/1.4962970

${ }^{73}$ www.seattletimes.com/opinion/editorials/another-nobel-prize-for-uw-scientist-illustratesvalue-of-pure-science

${ }^{74}$ McLuhan, M. (1964). Understanding Media: The Extensions of Man.

http://robynbacken.com/text/nw research.pdf

${ }^{75}$ Krugman, P. (1979). A model of innovation, technology transfer, and the world distribution of income. Journal of Political Economy, 253-266.

http://pioneer.netserv.chula.ac.th/ kkornkar/inter $\% 20 \operatorname{trade} \% 20$ course $/ \mathrm{a} \% 20$ model $\% 20 \mathrm{of} \% 20 \mathrm{In}$ novation.pdf

${ }^{76} \mathrm{http}: / / \mathrm{xml}$.coverpages.org/PML-MIT-AUTOID-WH-005.pdf

${ }^{77}$ Shapiro, C. and H. R. Varian (1999). Information rules: A strategic guide to the network economy. Harvard Business Press. http://faculty.csuci.edu/minder.chen/MBA550/reading/halvarian-information-rules-chapter-1.pdf

${ }^{78} \mathrm{http}: / /$ www.nber.org/papers/w23684

79 http://www.itsoc.org/resources/Shannon-Centenary/shannon-work-legacy-paper

${ }^{80}$ Greenstein, S. and F. Nagle (2014). Digital dark matter and the economic contribution of Apache. Research Policy 43(4), 623-631.

https://pdfs.semanticscholar.org/32ff/6098dc5d26551107935b78ef4a34f0b9aece.pdf

${ }^{81} \mathrm{http}: / /$ autoid.mit.edu/iot research initiative

${ }^{82}$ http://odl.mit.edu/ 